\title{
POLYTOPES AND LARGE COUNTEREXAMPLES
}

\author{
PER ALEXANDERSSON
}

\begin{abstract}
In this short note, we give large counterexamples to natural questions about certain order polytopes, in particular, Gelfand-Tsetlin polytopes. Several of the counterexamples are too large to be discovered via a brute-force computer search.

We also show that the multiset of hooks in a Young diagram is not enough information to determine the Ehrhart polynomial for an associated order polytope. This is somewhat counter-intuitive to the fact that the multiset of hooks always determine the leading coefficient of the Ehrhart polynomial.
\end{abstract}

\section{INTRODUCTION}

It is nowadays easy to make conjectures based on extensive computer evidence. This note gives several examples where the brute-force approach yield strong support for natural conjectures, but turns out to fail in higher dimensions.

There is a long-standing open conjecture regarding Kostka numbers, with plenty of computational support. It is conjectured, KTT04] that the map $k \rightarrow K_{k \lambda / k \mu, k w}$ is a polynomial with non-negative coefficients. In particular, this conjecture implies that the Ehrhart polynomial of the Birkhoff polytopes (defined as the convex hull of all $n \times n$ permutation matrices) has non-negative coefficients - also an open problem.

The Kostka coefficients can be computed by counting the lattice points in the intersection of a hyperplane defined by $w$ and the Gelfand-Tsetlin polytope $\mathcal{G}_{\lambda / \mu}$. It is therefore natural to study the Ehrhart polynomial of other restrictions of $\mathcal{G}_{\lambda / \mu}$. In Example 2, we give an example of a face of a certain $\mathcal{G}_{\lambda / \mu}$ with a negative coefficient. The example is too large to be discovered by brute force computer search, suggesting that eventual counterexamples to the Koskta numbers conjecture is of a similar nature.

One can show that the Gelfand-Tsetlin polytopes $\mathcal{G}_{\lambda / \mu}$ have the integer decomposition property (IDP), and that certain intersections with hyperplanes also give rise to this property, see Ale16. We present a large (carefully constructed) counterexample to a natural conjecture in this setting.

A few recent papers present conjectures regarding the IDP and Ehrhart coefficients, see [BDS16] and [HOT16]. The latter regards lecture hall polytopes, which are closely related to the polytopes we consider below in Example 3, suggesting that possible counterexamples to conjectures in [HOT16] only occur in high dimensions.

2010 Mathematics Subject Classification. 52B12, 05E10. 
Specifying the multiset of hooks gives very strong restrictions on a Young diagram, and therefore, a related order polytope. We show that the multiset of hooks of a Young diagram does not determine the Ehrhart polynomial of the order polytope - even though the multiset determines the leading coefficient. A similar example is provided for trees.

1.1. Order polytopes. We follow the terminology in [Sta86, Sta11]. Given a partial order $P$ on the set $[n]$, we associate a polytope $\mathcal{O}(P)$, called the order polytope of $P$. It is the polytope in $\mathbb{R}^{n}$ defined via the inequalities

$$
x_{i} \leq x_{j} \text { if } i<_{P} j \quad \text { and } \quad 0 \leq x_{i} \leq 1 \text { for } 1 \leq i \leq n .
$$

There is a slight generalization of order polytopes, called marked order polytopes, see e.g., ABS11, where we replace the conditions $0 \leq x_{i} \leq 1$ with $a_{i} \leq x_{i} \leq b_{i}$ for $1 \leq i \leq n$, where the $a_{i}$ and $b_{i}$ are fixed constants. We will mostly be concerned with a special case of marked order polytopes, the Gelfand-Tsetlin polytopes, which we define below.

One property worth mentioning is that all marked order polytopes with integer boundary conditions $a_{i}$ and $b_{i}$, are integral, i.e., all vertices are integer lattice points. In fact, integral marked order polytopes admit a unimodular triangulation - a triangulation into simplices with integer vertices, all having normalized volume 1. To sketch a proof of the latter statement, suppose that the polytope is in $\mathbb{R}^{n}$ and consider the hyperplanes

$$
x_{i}=m \text { and } x_{i}=x_{j}+m \text { for all } 1 \leq i, j \leq n \text { and } m \in \mathbb{Z} .
$$

The cube with vertices in $\{0,1\}^{n}$ is partitioned into $n$ ! unimodular simplices by these hyperplanes, implying that (2) determines a unimodular triangulation of $\mathbb{R}^{n}$. Finally note that the defining hyperplanes of a marked order polytope is a subset of the hyperplanes in (2).

1.2. Gelfand-Tsetlin polytopes. Gelfand-Tsetlin polytopes were first introduced in GT50. Consider a parallelogram arrangement of non-negative numbers,

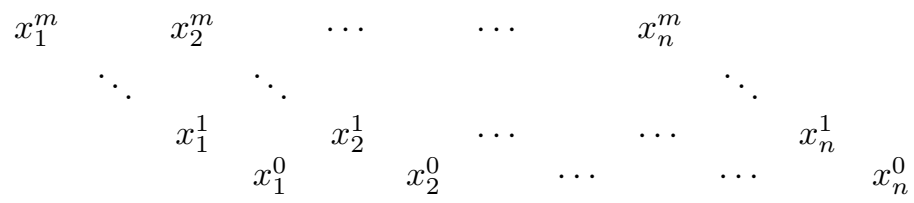

where the entries must satisfy the inequalities

$$
x_{j}^{i+1} \geq x_{j}^{i} \text { and } x_{j}^{i} \geq x_{j+1}^{i+1}
$$

for all values of $i, j$ where the indexing is defined. Note that horizontal rows and down-right diagonals are weakly decreasing, while down-left diagonals are weakly increasing.

Let $\lambda \supset \mu$ be partitions and fix the top and bottom rows in the diagram to be $\lambda$ and $\mu$, i.e., $x_{i}^{m}=\lambda_{i}$ and $x_{i}^{0}=\mu_{i}$ for $1 \leq i \leq n$. These conditions together with the inequalities in Eq. (3) defines a polytope $\mathcal{G}_{\lambda / \mu}$. Note that this polytope also depends on the choice of $m$. One can show that lattice points in $\mathcal{G}_{\lambda / \mu}$ are in 
bijection with semi-standard Young tableaux with shew shape $\lambda / \mu$ and entries in $[m]$, see e.g., Sta01].

In the case $\mu$ is the empty partition, many of the entries in the parallel arrangement are forced to be 0 . It suffices to keep track of the triangular pattern

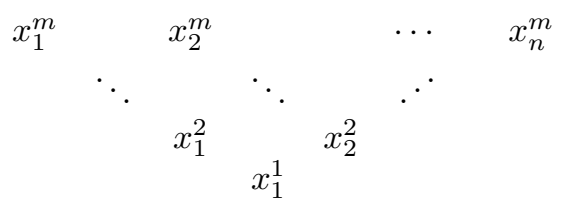

where the top row is fixed to be $\lambda$. We denote this polytope $\mathcal{G}_{\lambda}$.

1.3. Ehrhart polynomial. Given a polytope $\mathcal{P} \subset \mathbb{R}^{n}$ with integer vertices, the Ehrhart polynomial $\operatorname{ehr}_{\mathcal{P}}(n)$ is the function defined as

$$
\operatorname{ehr}_{\mathcal{P}}(n)=\#\left(n \mathcal{P} \cap \mathbb{Z}^{n}\right) .
$$

In other words, $\operatorname{ehr}_{\mathcal{P}}(n)$ counts the number of lattice points in the $n$th dilate of $\mathcal{P}$. Ehrhart showed that this is a polynomial in $n$.

We will use the notation $\operatorname{ehr}_{\lambda / \mu}(n)$ or simply $\operatorname{ehr}_{\lambda}(n)$ to denote the Ehrhart polynomials associated with $\mathcal{G}_{\lambda / \mu}$ and $\mathcal{G}_{\lambda}$, respectively. One can show that

$$
\operatorname{ehr}_{\lambda / \mu}(n)=\mathrm{s}_{n \lambda / n \mu}\left(1^{m}\right)
$$

where $\mathrm{s}_{\lambda / \mu}(\mathbf{x})$ is the skew Schur function and $1^{m}=(1,1, \ldots, 1)$ with $m$ ones and $n \lambda$ is interpreted as elementwise multiplication. Remember, $m+1$ is the number of rows in the parallelogram arrangement defining the Gelfand-Tsetlin polytope.

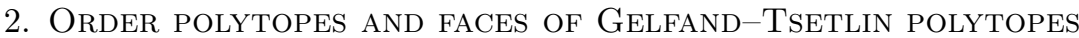

Now we are ready to give the first counterexample to a reasonable conjecture. An exhaustive search on the computer verifies that all order polytopes with dimension $\leq 7$ have no negative coefficients in their Ehrhart polynomials.

Example 1 (Negative Ehrhart coefficients). There are order polytopes with negative Ehrhart coefficients. Consider the following polytope, given by the inequalities $0 \leq z \leq x_{i} \leq 1$ for $i=1, \ldots, \ell$ : It is fairly straightforward to show that this order

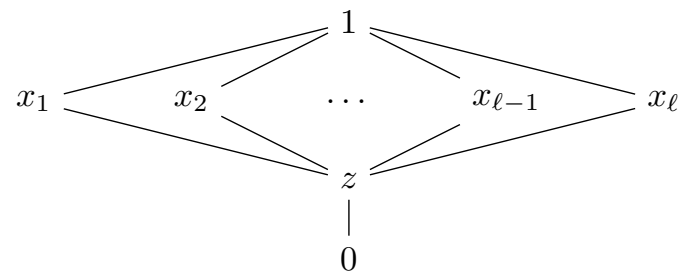

Figure 1 . For $\ell=20$, there are negative coefficients. 
polytope has Ehrhart polynomial given by $\operatorname{ehr}(n)=\sum_{j=1}^{n+1} j^{\ell}$. This expression can be evaluated using a computer algebra system and for $\ell=20$ we get

$$
\operatorname{ehr}(n)=\frac{1}{6930}\left(6930-3528231 n+1316700 n^{2}+32027050 n^{3}+\cdots\right) .
$$

For $\ell<20$, all coefficients are non-negative. Note that even using state-of-the-art lattice point counting software such as lattE to determine the Ehrhart polynomial of this order polytope is out of reach due to the high dimension.

Note: This construction more or less appear in [Sta11, Figure 3.87] and should be attributed to R. Stanley Sta15].

It is easy to see from the Weyl character formula that for a non-skew shape $\lambda$, the Ehrhart polynomial $\operatorname{ehr}_{\lambda}(n)$ has non-negative coefficients. A natural question is then to consider faces of Gelfand-Tsetlin polytopes.

Faces of Gelfand-Tsetlin polytopes are obtained by forcing some of the inequalities in Eq. (3) to be equalities. Such faces appear in many places in representation theory and algebraic combinatorics. For example, certain faces are responsible for generating key polynomials, see KST12, and they are also in correspondence with so called $r c$-graphs and pipe dreams, see KM05. Flagged Schur polynomials can be computed as a sum over lattice points in certain faces of Gelfand-Tsetlin polytopes.

We can use the previous example to construct a face of a Gelfand-Tsetlin polytope $\mathcal{G}_{\lambda}$, such that a certain face has a negative coefficient in the Ehrhart polynomial.

Example 2. We can construct a one-parameter family of faces of Gelfand-Tsetlin polytopes with top row given by $\lambda=\left(1^{\ell}, 0^{\ell+1}\right)$ :

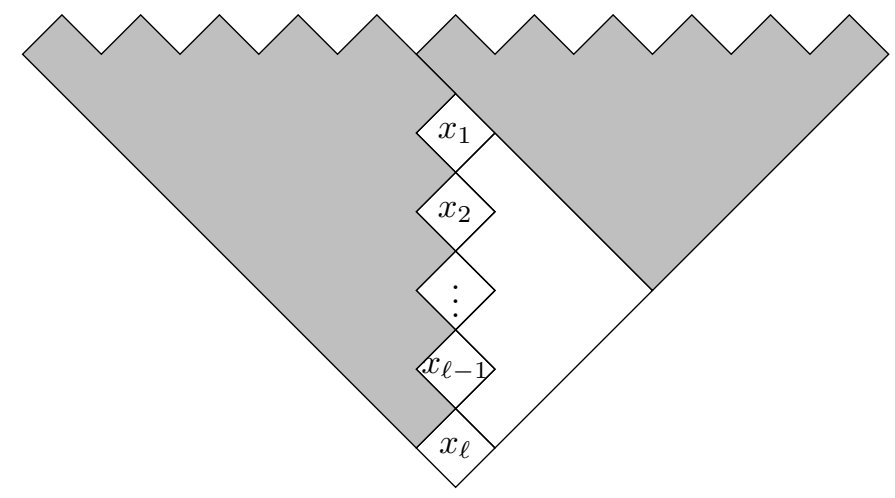

There are two shaded regions and one larger unshaded region, where all entries within each respective region are all forced to be equal. These conditions determine a face. Note that all entries within the leftmost shaded region are all equal to 1 , while all variables in the rightmost region are equal to 0 . Lattice points in this face are in clear bijection with lattice points in the order polytope in Example 1, so for $\ell=20$, the Ehrhart polynomial has a negative coefficient. 


\section{Partition polytope And a large GT COUnterexample}

As in the previous section, we first construct a counterexample in a simpler setting, which is then transferred to a statement about Gelfand-Tsetlin polytopes. The property we consider in this section is the integer decomposition property.

An integral polytope $\mathcal{P} \subset \mathbb{R}^{d}$ is said to have the integer decomposition property (IDP) if for every positive integer $k$ and $\mathbf{x} \in k \mathcal{P} \cap \mathbb{Z}^{d}$, we can find $\mathbf{x}_{1}, \mathbf{x}_{2}, \ldots, \mathbf{x}_{k} \in$ $\mathcal{P} \cap \mathbb{Z}^{d}$ such that $\mathbf{x}_{1}+\cdots+\mathbf{x}_{k}=\mathbf{x}$. One can show the following series of implications for a polytope $\mathcal{P}$;

$\mathcal{P}$ has a unimodular triangulation $\Longrightarrow \mathcal{P}$ has the IDP $\Longrightarrow \mathcal{P}$ is integral.

Let $\mathcal{P}_{a, b}$ denote the convex cull of all partitions of $a$ with $b$ parts, seen as integer points in $\mathbb{R}^{b}$. Computer experiments suggests that $\mathcal{P}_{a, b}$ has the IDP if $a \leq 18$ and $b \leq 9$. However, we have the following counterexample, which was pointed out by Robert Davis to the author via private communication:

Example 3. The polytope $\mathcal{P}_{18,9}$ does not have the IDP. Consider the lattice point $p=(6,6,6,6,4,4,2,1,1)$. It is straightforward to verify that

$$
\begin{aligned}
p= & \frac{1}{2}(4,4,4,4,1,1,0,0,0)+\frac{1}{2}(3,3,3,3,3,3,0,0,0)+ \\
& \frac{1}{2}(3,3,3,3,2,2,2,0,0)+\frac{1}{2}(2,2,2,2,2,2,2,2,2)
\end{aligned}
$$

so it is clear that $p \in 2 \cdot \mathcal{P}_{18,9}$. However, an exhaustive search on the computer shows that $p$ cannot be expressed as a sum of two lattice points in $\mathcal{P}_{18,9}$.

The point $p$ above is not the only point in $2 \cdot \mathcal{P}_{18,9}$ that cannot be expressed as a sum of two integer points in $\mathcal{P}_{18,9}$ - another such point is $(6,6,5,5,5,4,2,2,1)$.

Note that each row in a Gelfand-Tsetlin pattern is a partition. Let us add an extra restriction on Gelfand-Tsetlin polytopes, and let $\mathcal{G}_{\lambda / \mu, w}$ denote the polytope consisting of all points satisfying Eq. (3), with the additional condition that the entries in row $i$ sum up to $w_{i}$. Here, $w$ is an integer vector with $m$ rows. The polytopes $\mathcal{G}_{\lambda / \mu, w}$ are in general no longer integral, a fact first pointed out in KTT04, and further extended in LM04, LM06, Ale16].

Since Gelfand-Tsetlin polytopes have nice properties with respect to unimodular triangulations and the IDP, it is natural to ask if similar properties hold for $\mathcal{G}_{\lambda / \mu, w}$. In fact, in [Ale16] we show that $\mathcal{G}_{\lambda / \mu, 1^{m}}$ always has a unimodular triangulation whenever it is integral.

Can we do something in the non-integral case? By using Example 3 as a starting point, we can show the following statement:

Proposition 4. Let $\mathcal{G}_{\lambda / \mu, w}^{*}$ be the polytope defined as the convex hull of the integer points in $\mathcal{G}_{\lambda / \mu, w}^{*}$. Then there is a $\mathcal{G}_{\lambda / \mu, w}^{*}$ which does not satisfy the integer decomposition property.

We have not been able to find a $\mathcal{G}_{\lambda / \mu, w}^{*}$ which does not have the IDP by brute force computer search. The following is the smallest known counterexample which has been carefully constructed: 
Example 5. Let

$$
\begin{aligned}
\lambda & =(4,4,4,4,3,3,2,2,2), \quad \mu=(2,0,0,0,0,0,0,0,0) \\
w & =(2,4,5,6,7,8,9,10,11,12,14,16,18,20,22,23,25,27,28) .
\end{aligned}
$$

Then $\mathcal{G}_{\lambda / \mu, w}^{*}$ does not have the IDP. Consider the following GT-pattern $G$ :

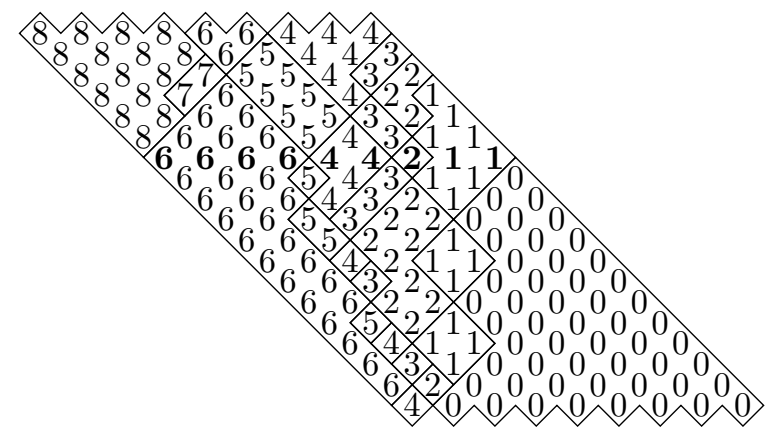

We can verify that $G$ is lattice point in $2 \cdot \mathcal{G}_{\lambda / \mu, w}^{*}$, since $G=\frac{1}{2}\left(G_{1}+G_{2}+G_{3}+G_{4}\right)$, where $G_{1}, \ldots, G_{4} \in \mathcal{G}_{\lambda / \mu, w}^{*}$ are given by

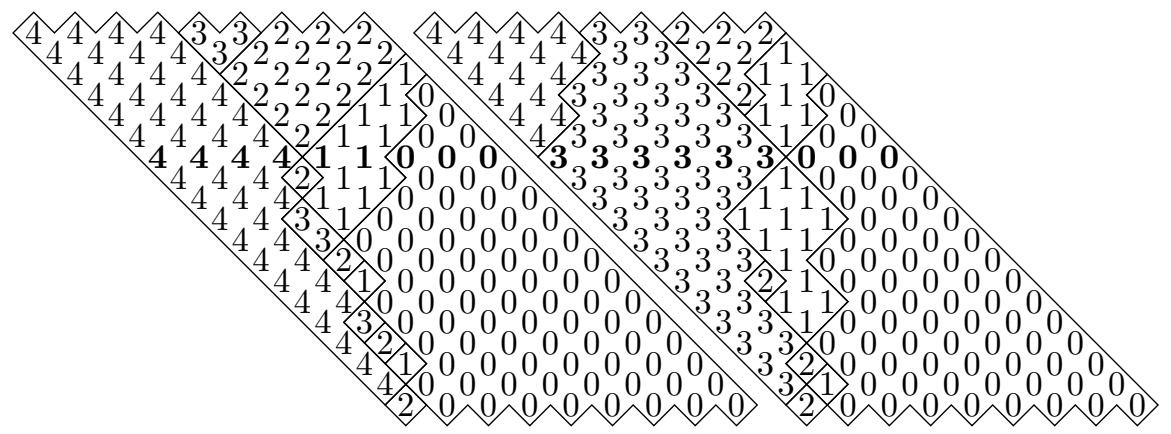

and

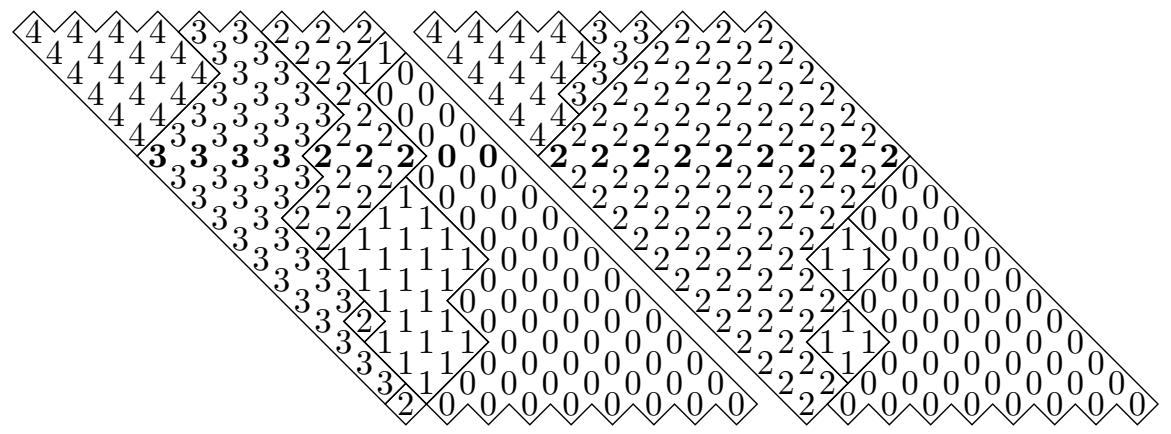

We cannot write $G$ as as sum of two integral GT-patterns in $\mathcal{G}_{\lambda / \mu, w}^{*}$, since that would, in particular, express the partition in row 15 (entries in bold) as a sum of two only two integer partitions with size 18 and 9 parts. This is impossible due to Example 3.

Here is how the full example was constructed. The starting point is Example 3 , which guarantees that $G$ is not a sum of two GT-patterns in $\mathcal{G}_{\lambda / \mu, w}^{*}$. The idea is to 
extend the four partitions in Eq. (4) to GT-patterns. Note that there are several restrictions: the inequalities in Eq. (3) have to be satisfied and all four GT-patterns must have the same parameters $\lambda / \mu$ and $w$. Finally, $G_{1}+\cdots+G_{4}$ has to result in a GT-pattern with only even entries.

With these restrictions, starting from Example 3 and extending the partitions row by row by a computer search, eventually leads to the above example. Note that this approach is not guaranteed to work in general - using the other point, $(6,6,5,5,5,4,2,2,1)$ in $2 \cdot \mathcal{P}_{18,9}$ with a similar setup is impossible to extend to a full example.

Remark 6. It is known (see [Ras04]) that the polytope $\mathcal{G}_{\lambda / \mu, w}$ always has a polynomial Ehrhart functions, despite not being integral in general. Lattice points in $\mathcal{G}_{\lambda / \mu, w}$ are enumerated by the skew Kostka coefficient, $K_{\lambda / \mu, w}$. It is conjectured in KTT04 that the function $n \rightarrow K_{n \lambda / n \mu, n w}$ is a polynomial with non-negative coefficients. Equivalently, the conjecture states that $\mathcal{G}_{\lambda / \mu, w}$ has an Ehrhart polynomial with non-negative coefficients.

In the light of Example 2, it might be that eventual counterexamples to the Kostka coefficient conjecture only appear in extremely $(\geq 200)$ high dimensions.

\section{Hooks, volume And LATtice POINT ENUmeration}

We now leave the Gelfand-Tsetlin polytopes and focus on certain order polytopes.

Let $P$ be a partial order on $[n]$. A linear extension of $P$ is a permutation $\pi$ such that $\pi(i)<\pi(j)$ if $i<_{P} j$. Counting linear extensions of posets is \#P-hard in general, BW91 but for some posets there are efficient formulas.

Given a partition $\lambda$, we construct a poset $P_{\lambda}$ presenting its Hasse diagram as the Ferrers diagram of shape $\lambda$ rotated $135^{\circ}$, see Example 8 below where covering relations for two such posets are illustrated. Linear extensions of $P_{\lambda}$ correspond to standard Young tableaux of shape $\lambda$. Since $P_{\lambda}$ defines an order polytope, it follows from [Sta86] that the number of linear extensions of $P_{\lambda}$ is the same as the normalized volume of $\mathcal{O}\left(P_{\lambda}\right)$.

We know that the number of linear extensions of $P_{\lambda}$ can be computed using the Hook formula, [FdBRT54:

Theorem 7 (Hook formula).

$$
\#\left\{\text { linear extensions of } P_{\lambda}\right\}=\frac{n !}{\prod_{(i, j) \in \lambda} \operatorname{hook}_{\lambda}((i, j))} .
$$

We define the hook $\operatorname{hook}_{\lambda}((i, j))$ as $\lambda_{i}+\lambda_{j}^{\prime}-i-j+1$. The hooks appear as vertex labels in the diagrams in Example 8 .

Furthermore, the volume of a polytope is determined by the leading coefficient in its Ehrhart polynomial. It is therefore natural to ask if we can determine the entire Ehrhart polynomial of $\mathcal{O}\left(P_{\lambda}\right)$ from the multiset of hooks of the diagram $\lambda$. 
Example 8. Consider the diagrams $\lambda=(8,5,4)$ and $\mu=(7,7,2,1)$. These diagrams have the same multiset of hooks (indicated by vertex labels) and thus the volume of $\mathcal{O}\left(P_{\lambda}\right)$ and $\mathcal{O}\left(P_{\mu}\right)$ coincide.

(1)

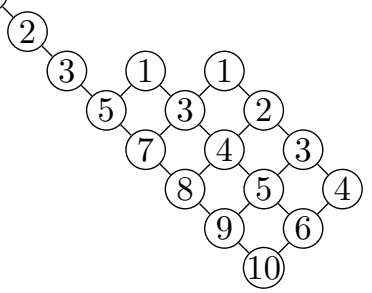

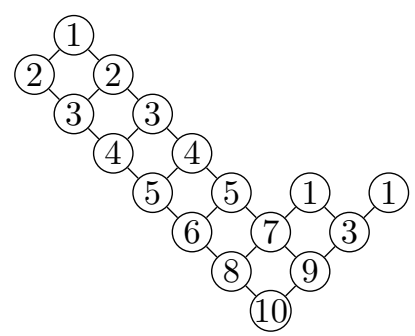

However, these polytopes have different Ehrhart polynomials, one can easily verify that $\operatorname{ehr}_{\mathcal{O}\left(P_{\lambda}\right)}(1)=115 \operatorname{but} \operatorname{ehr}_{\mathcal{O}\left(P_{\mu}\right)}(1)=134$. Using the software Normaliz, one can find the complete Ehrhart polynomials

$$
\begin{gathered}
\operatorname{ehr}_{\mathcal{O}\left(P_{\lambda}\right)}(k)=7 F(k)(k+3)(k+4)\left(4 k^{3}+35 k^{2}+101 k+90\right) \\
\operatorname{ehr}_{\mathcal{O}\left(P_{\mu}\right)}(k)=F(k)(k+6)(k+7)\left(28 k^{3}+161 k^{2}+301 k+180\right)
\end{gathered}
$$

where

$$
F(k)=\frac{(k+1)(k+2)^{2}(k+3)^{2}(k+4)^{2}(k+5)^{2}(k+6)(k+7)(k+8)}{36578304000} .
$$

One can ask if the multiset of hooks perhaps determine other properties of $\mathcal{O}\left(P_{\lambda}\right)$. In light off Example 8, it is rather surprising that for any $\lambda$, the Ehrhart polynomial of the restriction

$$
P_{\lambda} \cap\left\{\left(x_{1}, \ldots, x_{n}\right) \in \mathbb{R}^{n}: x_{1}+\ldots+x_{n}=1\right\}
$$

is determined by the multiset of hooks. This is proved in Pak01, Lemma 1], which restates the Hook Content Formula as an equality between lattice point cardinalities.

There is a similar hook formula for counting the number of linear extensions of trees, see [Knu98. Here, hook values are simply given by subtree size.

Example 9. The trees $T$ and $T^{\prime}$ have the same multiset of hooks but different Ehrhart polynomials. Each node $v$ is decorated by its hook value - the size of the subtree with root $v$.
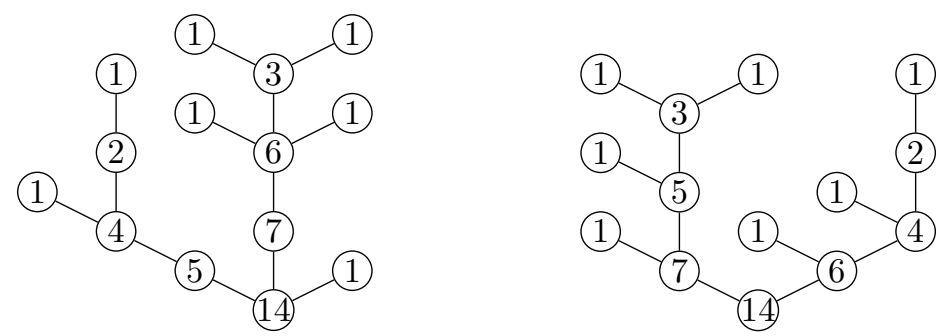
We have that

$$
\begin{aligned}
\operatorname{ehr}_{\mathcal{O}(T)}(n) & =F(n)\left(51480 n^{9}+1182984 n^{8}+11490017 n^{7}+61564083 n^{6}\right. \\
& +199510913 n^{5}+404186041 n^{4}+512043278 n^{3} \\
& \left.+393196652 n^{2}+167403432 n+30270240\right)
\end{aligned}
$$

and

$$
\begin{aligned}
\operatorname{ehr}_{\mathcal{O}\left(T^{\prime}\right)}(n) & =F(n)\left(51480 n^{9}+1173975 n^{8}+11327855 n^{7}+60383085 n^{6}\right. \\
& +195027707 n^{5}+394660980 n^{4}+500753090 n^{3} \\
& \left.+386259540 n^{2}+165675888 n+30270240\right)
\end{aligned}
$$

where $F(n)=\frac{(n+1)(n+2)(n+3)(n+4)(n+5)}{3632428800}$.

Note that it is enough to notice that $\operatorname{ehr}_{\mathcal{O}(T)}(1) \neq \operatorname{ehr}_{\mathcal{O}\left(T^{\prime}\right)}(1)$ to deduce that the Ehrhart polynomials are different. This computation can be done by brute force. To compute the full Ehrhart polynomial, more sophisticated software such as lattE is required.

By combining the result in [BW89] and the theory of $P$-partitions by Stanley Sta11, one obtains the analogous statement of (5) for trees.

Acknowledgement. The author would like to thank Greta Panova for pointing out reference Pak01] as well as for several insightful comments. We also thank Winfied Bruns for the computations in Normaliz.

This work has been funded by the Knut and Alice Wallenberg Foundation (2013.03.07).

\section{REFERENCES}

[ABS11] Federico Ardila, Thomas Bliem, and Dido Salazar, Gelfand-Tsetlin polytopes and Feigin-Fourier-Littelmann-Vinberg polytopes as marked poset polytopes, J. Comb. Theory, Ser. A 118 (2011), no. 8, 2454-2462.

[Ale16] Per Alexandersson, Gelfand-Tsetlin polytopes and the integer decomposition property, European Journal of Combinatorics 54 (2016), 1-20.

[BDS16] B. Braun, R. Davis, and L. Solus, Detecting the Integer Decomposition Property and Ehrhart Unimodality in Reflexive Simplices, ArXiv e-prints (2016).

[BW89] Anders Björner and Michelle L Wachs, q-Hook length formulas for forests, Journal of Combinatorial Theory, Series A 52 (1989), no. 2, 165 - 187.

[BW91] Graham Brightwell and Peter Winkler, Counting linear extensions, Order 8 (1991), no. $3,225-242$.

[FdBRT54] J.S. Frame, G. de B. Robinson, and R.M. Thrall, The hook graphs of the symmetric group, Canad. J. Math. 6 (1954), 316-325.

[GT50] I. M. Gelfand and M. L. Tsetlin, Finite dimensional representations of the group of unimodular matrices, Doklady Akad. Nauk SSSR (N.S.) (1950), 825-828, (Russian).

[HOT16] T. Hibi, M. Olsen, and A. Tsuchiya, Gorenstein properties and integer decomposition properties of lecture hall polytopes, ArXiv e-prints (2016).

[KM05] Mikhail Kogan and Ezra Miller, Toric degeneration of Schubert varieties and GelfandTsetlin polytopes, Advances in Mathematics 193 (2005), no. 1, 1- 17.

[Knu98] Donald E. Knuth, The art of computer programming, volume 3: (2nd ed.) sorting and searching, Addison Wesley Longman Publishing Co., Inc., Redwood City, CA, USA, 1998.

[KST12] Valentina A Kirichenko, Evgeny Yu Smirnov, and Vladlen A Timorin, Schubert calculus and Gelfand-Zetlin polytopes, Russian Mathematical Surveys 67 (2012), no. 4, 685 . 
[KTT04] R.C. King, C. Tollu, and F. Toumazet, Stretched Littlewood-Richardson coefficients and Kostka coefficients, CRM Proceedings and Lecture Notes (2004), no. 34, 99-112.

[LM04] Jesús A. De Loera and Tyrrell B. McAllister, Vertices of Gelfand-Tsetlin Polytopes, Discrete \& Computational Geometry 32 (2004), no. 4, 459-470.

[LM06] On the Computation of Clebsch-Gordan Coefficients and the Dilation Effect, Experimental Mathematics 15 (2006), no. 1, 7-19.

[Pak01] Igor Pak, Hook length formula and geometric combinatorics., Séminaire Lotharingien de Combinatoire [electronic only] 46 (2001), B46f, 13 p., electronic only-B46f, 13 p., electronic only (eng).

[Ras04] Etienne Rassart, A polynomiality property for Littlewood-Richardson coefficients, J. Comb. Theory Ser. A 107 (2004), no. 2, 161-179.

[Sta86] Richard P. Stanley, Two poset polytopes, Discrete \& Computational Geometry 1 (1986), no. 1, 9-23.

[Sta01] Richard P. Stanley, Enumerative Combinatorics: Volume 2, 1st ed., Cambridge University Press, 2001.

[Sta11] _ Enumerative Combinatorics: Volume 1, 2nd ed., Cambridge University Press, 2011.

[Sta15] - Positivity of Ehrhart polynomial coefficients, MathOverflow, 32 2015, URL:http://mathoverflow.net/q/200574 (version: 2015-03-20).

Dept. of Mathematics, University of Pennsylvania. Philadelphia, PA

E-mail address: per.w.alexandersson@gmail.com 